\title{
Double-layer ion acceleration triggered by ion magnetization in expanding radiofrequency plasma sources
}

\author{
Kazunori Takahashi, ${ }^{1,2, a)}$ Christine Charles, ${ }^{2}$ Rod W. Boswell, ${ }^{2}$ and Tamiya Fujiwara ${ }^{1}$ \\ ${ }^{1}$ Department of Electrical and Electronic Engineering, Iwate University, Morioka 020-8551, Japan \\ ${ }^{2}$ Space Plasma, Power and Propulsion Group, Research School of Physics and Engineering, The Australian \\ National University, Canberra 0200, Australia
}

(Received 20 May 2010; accepted 21 September 2010; published online 7 October 2010)

\begin{abstract}
Ion energy distribution functions downstream of the source exit in magnetically expanding low-pressure plasmas are experimentally investigated for four source tube diameters ranging from about 5 to $15 \mathrm{~cm}$. The magnetic-field threshold corresponding to a transition from a simple expanding plasma to a double layer-containing plasma is observed to increase with a decrease in the source tube diameter. The results demonstrate that for the four geometries, the double layer and the accelerated ion beam form when the ion Larmour radius in the source becomes smaller than the source tube radius, i.e., when the ions become magnetized in the source tube. (0) 2010 American Institute of Physics. [doi:10.1063/1.3499653]
\end{abstract}

Electric double layer (DL) formation in magnetically expanding, low-pressure laboratory plasmas has been active research topic associated with particle acceleration in space plasmas ${ }^{1}$ and the development of electrodeless plasma propulsion devices. ${ }^{2}$ Once the DL is formed, ions in the source are electrostatically accelerated by the DL potential drop and the resulting high-velocity ion beam can be detected in a diffusion chamber downstream of the DL. ${ }^{3}$ The beam velocity and density have been characterized for various experimental conditions, e.g., gas pressure and gas type, ${ }^{4,5} \mathrm{rf}$ frequencies for plasma production, ${ }^{6}$ and magnetic-field strengths and configurations in various machines. ${ }^{7-11} \mathrm{Al}-$ though a previously reported analytical $\operatorname{model}^{4}$ and onedimensional (1D) particle-in-cell (PIC) simulation ${ }^{12}$ have described some aspects of the DL behavior, these have not included the role of the expanding magnetic fields. Recently, a transition from a simple expanding plasma to a DLcontaining plasma has been experimentally observed when increasing the source magnetic field with a threshold at about $50 \mathrm{G}$ in a 15 -cm-diameter source tube. ${ }^{7}$ It was postulated that this threshold would correspond to the magnetization of the ions in the plasma source. Identifying the mechanism behind the threshold in the magnetic field at the transition is of interest for the development of two-dimensional (2D) analytical or PIC models.

In the present letter, it is experimentally demonstrated for an extended range of source diameters $(\sim 5-15 \mathrm{~cm})$ that the DLs and the subsequent ion beams are generated when the ion Larmour radius in the source becomes smaller than the radius of the source tube.

Experiments with the two smaller source tube diameters $(4.6$ and $6.5 \mathrm{~cm})$ are performed in the ElectroMagnets expanding Plasma machine at Iwate University (EMPI) shown in Fig. $1,{ }^{13}$ and experiments with the two larger source tube diameters $(9.2$ and $13.7 \mathrm{~cm}$ ) are performed in the previously described "Chi-Kung" device at the Australian National University (ANU). ${ }^{14}$ The tube length is in the $26-31 \mathrm{~cm}$ range. In all four geometries, the rapid potential drop of the DL has been observed near the source exit and the ion beam accel-

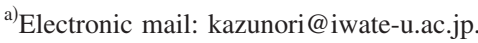

erated by the DL has been detected in the downstream side of the DL. ${ }^{3}$ EMPI has a 17.5 -cm-long and 20.8 -cm-diameter grounded diffusion chamber, and Chi-Kung has a 30-cmlong and 32-cm-diameter grounded chamber. The EMPI system of Fig. 1 is pumped down to a base pressure below $10^{-5}$ Torr. The argon gas is introduced from the upstream flange of the source tube and the operating pressure is maintained in the $P_{\mathrm{Ar}}=0.4-0.6 \mathrm{mTorr}$ range. An argon plasma is excited by a triple-turn loop antenna located at $z=-16 \mathrm{~cm}$, wound around the source tube, and powered from a 13.56 $\mathrm{MHz}$ and $200 \mathrm{~W}$ rf generator through a matching circuit, where $z=0$ is defined as the source exit. The plasma is terminated by an insulator plate at $z=-25 \mathrm{~cm}$ at the upstream side. Two solenoid coils situated at $z=-23.2$ and $-8.7 \mathrm{~cm}$ provide divergent magnetic fields. Calculated fields for solenoid currents $I_{\mathrm{B}}=1,3$, and $5 \mathrm{~A}$ are plotted as solid lines in Fig. 2(a) together with the field measured for $I_{\mathrm{B}}=3$ A using a Gaussmeter. The magnetic-field lines are divergent near the source exit and the averaged magnetic-field strength in the

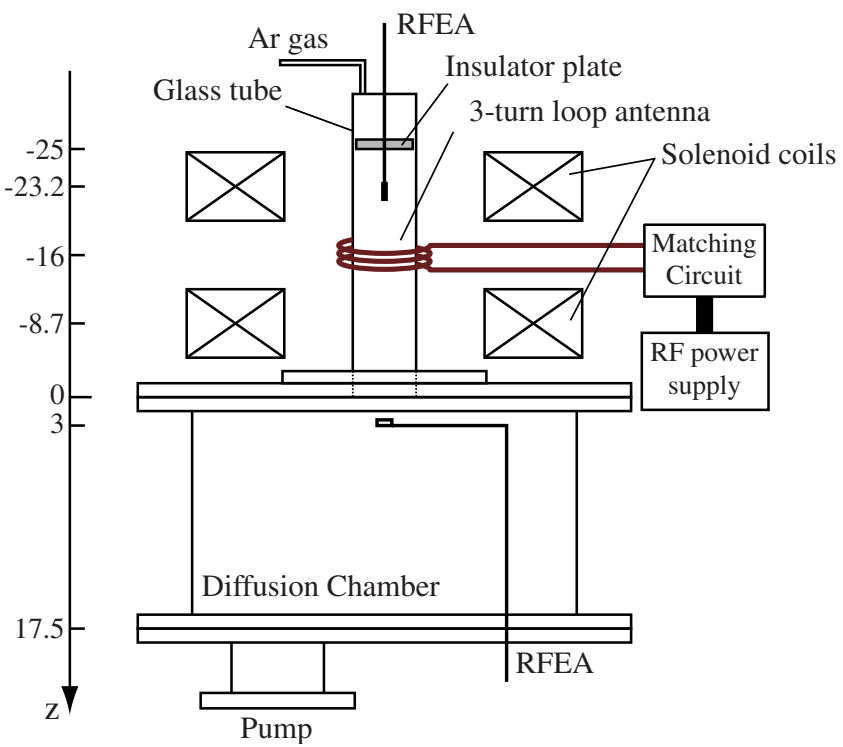

FIG. 1. (Color online) Schematic of EMPI with $4.6 \mathrm{~cm}$ or $6.5 \mathrm{~cm}$ diameter source tube. 
(a)

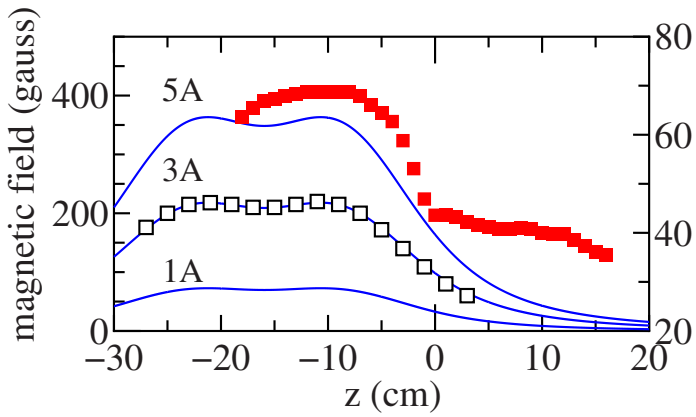

(b)

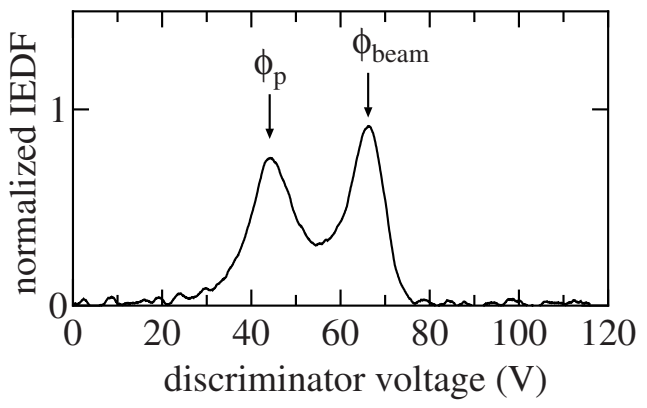

FIG. 2. (Color online) (a) Calculated magnetic-field strength (solid lines) for $I_{\mathrm{B}}=1,3$, and $5 \mathrm{~A}$ in $E M P I$, together with the measured magnetic fields (open squares) for $I_{\mathrm{B}}=3$ A. The filled squares shows the local plasma potential $\phi_{\mathrm{p}}$ for $P_{\mathrm{Ar}}=0.4 \mathrm{mTorr}, I_{\mathrm{B}}=5 \mathrm{~A}, P_{\mathrm{RF}}=200 \mathrm{~W}$, and the $6.5-\mathrm{cm}$-diameter source tube. (b) Normalized IEDF at $z=3 \mathrm{~cm}$ for the same operating conditions as filled squares in (a).

source tube for unit solenoid current is about $70 \mathrm{G} / \mathrm{A}$.

In EMPI, an axially movable retarding field energy analyzer (RFEA) facing the radial wall and inserted from the top of the source tube gives an axial profile of the local plasma potential $\phi_{\mathrm{p}}$. The filled squares in Fig. 2(a) show the local plasma potential $\phi_{\mathrm{p}}$ for $P_{\mathrm{Ar}}=0.4 \mathrm{mTorr}, I_{\mathrm{B}}=5 \mathrm{~A}$, and the $6.5-\mathrm{cm}$-diameter source tube. It is found that the rapid potential drop of the DL exists near the source exit. The measurement of the axial ion energy distribution function (IEDF) downstream of the DL is carried out at $z=3 \mathrm{~cm}$ using the RFEA facing the source tube. Figure 2(b) shows the normalized IEDF at $z=3 \mathrm{~cm}$ for the same operating conditions as the filled squares in Fig. 2(a), and clearly indicates the existence of the ion beam at a beam potential $\phi_{\text {beam }} \sim 66 \mathrm{~V}$ in addition to the thermal ions at $\phi_{\mathrm{p}} \sim 44 \mathrm{~V}$. The beam potential agrees well with the local plasma potential measured at the upstream edge of the DL near $z=-5 \mathrm{~cm}$. Hence, the accelerated ions are found to originate from the upstream edge of the DL and here the ion beam will be used as a marker of the DL formation.

Figure 3 shows the contour plots of the normalized IEDF for the (a) 13.7-cm-diameter source in Chi-Kung, (b) 6.5-cmdiameter, and (c) 4.6-cm-diameter sources in EMPI, as a function of the averaged magnetic-field strength in the source tube. Here, the operating conditions are chosen as (a) $P_{\mathrm{RF}}=550 \mathrm{~W}$ and $P_{\mathrm{Ar}}=0.3 \mathrm{mTorr}$; (b) and (c) $P_{\mathrm{RF}}=200 \mathrm{~W}$ and $P_{\mathrm{Ar}}=0.6 \mathrm{mTorr}$, respectively. The local plasma potential and the beam potential are also plotted as open circles and squares, respectively. As previously reported in Ref. 7, the single-peaked IEDFs indicating only the thermal ions are observed for low magnetic-field strength in all four geometries, while the double-peaked IEDFs including the accelerated ions are detected above thresholds of the magnetic field. Here, it is noted that the observed thresholds of the magnetic field at the transition from the single-peaked to double- (a)

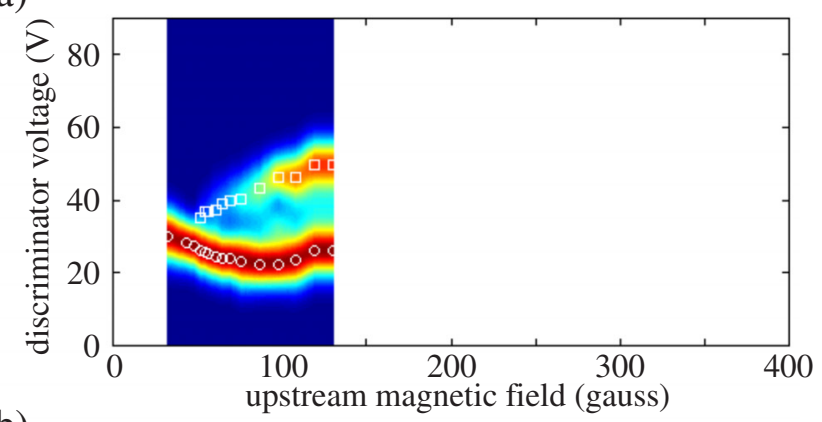

(b)

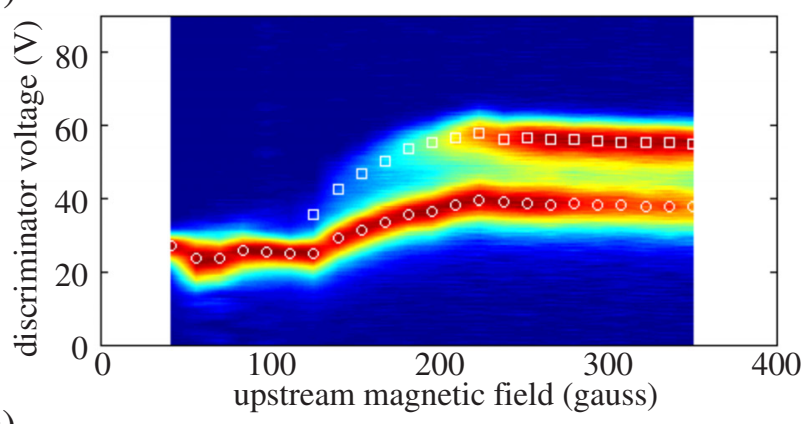

(c)

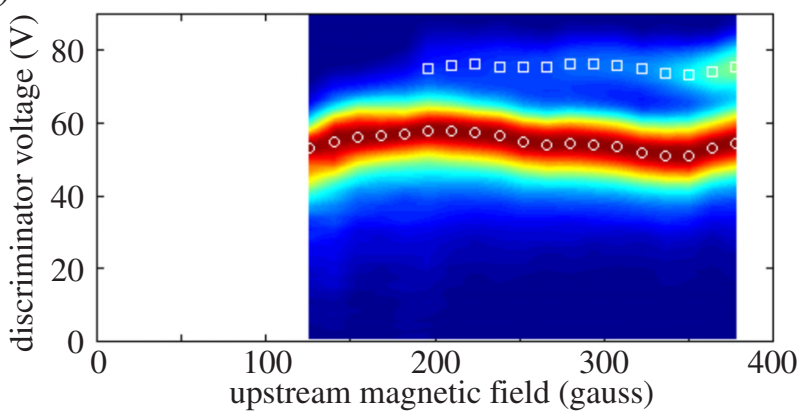

FIG. 3. (Color online) The normalized IEDFs for (a) $P_{\mathrm{Ar}}=0.3 \mathrm{mTorr}$ and $P_{\mathrm{RF}}=550 \mathrm{~W}$ in Chi-Kung with the 13.7-cm-diameter source, and for $P_{\mathrm{Ar}}$ $=0.6 \mathrm{mTorr}$ and $P_{\mathrm{RF}}=200 \mathrm{~W}$ in EMPI with the (b) $6.5-\mathrm{cm}$-diameter and (c) 4.6-cm-diameter source tubes, together with the local plasma potential $\phi_{\mathrm{p}}$ (open circles) and the beam potential $\phi_{\text {beam }}$ (open squares). The data of (a) is from Ref. 7.

peaked IEDFs are about (a) $50 \mathrm{G}$ for $13.7 \mathrm{~cm}$ source, (b) 125 $\mathrm{G}$ for $6.5 \mathrm{~cm}$ source, and (c) $195 \mathrm{G}$ for $4.6 \mathrm{~cm}$ source, respectively. The results of the $9.2 \mathrm{~cm}$-diameter source in $\mathrm{Chi}$ Kung for $P_{\mathrm{RF}}=250 \mathrm{~W}$ and $P_{\mathrm{Ar}}=0.3 \mathrm{mTorr}$ and $0.6 \mathrm{mTorr}$ are similar to Fig. 3(a), and are not shown here for clarify; they correspond to a transition at about $70 \mathrm{G}$.

The minimum upstream magnetic field threshold $B_{\mathrm{t}(\text { measured) }}$ corresponding to the transition from the single- to double-peaked IEDFs, which is the marker for the transition from the simple expanding plasma to the DL-containing expanding plasma, is plotted in Fig. 4 as a function of the inverse of the source radius $r_{\text {source }}$. The open triangle, filled square, and open square in Fig. 4 are obtained from Fig. 3 . The results for $P_{\mathrm{Ar}}=0.35 \mathrm{mTorr}$ and $0.6 \mathrm{mTorr}$ in the ANU device with the 9.2 -cm-diameter source tube are also plotted as filled and open circles, respectively, to verify that the change of pressure from about 0.3 to $0.6 \mathrm{mTorr}$ does not significantly alter the magnetic field threshold $B_{\mathrm{t}(\text { measured) }}$. It is found that $B_{\mathrm{t} \text { (measured) }}$ is nearly proportional to $1 / r_{\text {source }}$. The ion Larmour radius $r_{\mathrm{Li}}$ is now calculated as $r_{\mathrm{Li}}=v_{\mathrm{i}} / \omega_{\mathrm{ci}}$ as a function of the magnetic-field strength $B_{\mathrm{t}(\text { calculated) }}$, where $v_{\mathrm{i}}$ and $\omega_{\mathrm{ci}}$ are the ion thermal velocity and the ion cyclotron 


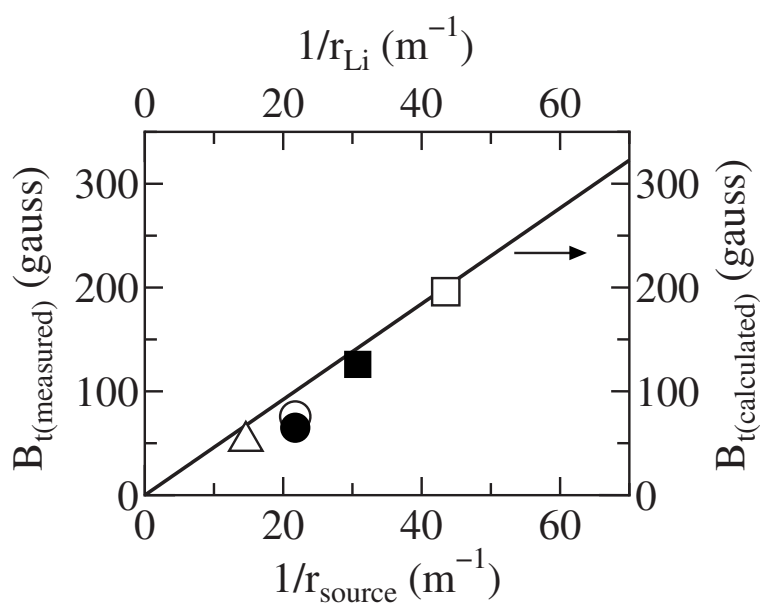

FIG. 4. Measured magnetic field $B_{\mathrm{t} \text { (measured) }}$ at the transition for DL formation vs the inverse of the source radius $r_{\text {source }}$ for the four geometries [internal source diameter of $13.7 \mathrm{~cm}$ (open triangle), $9.2 \mathrm{~cm}$ (open and filled circles), $6.5 \mathrm{~cm}$ (filled square), and $4.6 \mathrm{~cm}$ (open square)], and the calculated magnetic field $B_{\mathrm{t}(\text { calculated) }}$ vs the inverse in the ion Larmour radius $r_{\mathrm{Li}}$ (solid line).

frequency, respectively. The upstream ion temperature of 0.2 $\mathrm{eV}$ previously measured by laser induced fluorescence in the ANU device ${ }^{15}$ is used for the calculation of the ion thermal velocity. The calculated $B_{\mathrm{t}(\text { calculated })}$ is plotted versus the inverse $1 / r_{\mathrm{Li}}$ of the ion Larmour radius in Fig. 4 as a solid line. The results of Fig. 4 show that for the four geometries investigated here, the measured and calculated magnetic field threshold $B_{\mathrm{t}}$ agree surprisingly well. Hence the DL and the subsequent supersonic ion beam are spontaneously formed when the ion Larmour radius becomes smaller than the source tube radius; $r_{\mathrm{Li}}<r_{\text {source }}$. It has been previously shown that the reduced radial ion loss results in an increase in both the source density and the density gradient at the exit of the source tube. 7 A "mimic" of the latter was obtained in the previously reported 1D PIC simulation of the DL, ${ }^{12}$ by substituting the divergent magnetic field by a downstream particle loss scheme, where the loss frequency in the upstream source was chosen as zero to "trigger" the simulated DL. 2D simulation or analytical models would need to be developed for a better description of the DL mechanism and the present geometrical study provides a clear indication that the source magnetic field and its gradient near the source exit are critical parameters for DL formation.

In summary, the ion energy distributions and the plasmapotential structures are experimentally investigated in magnetically expanding plasmas for four plasma source geometries ranging from 4.6 to $13.7 \mathrm{~cm}$ in internal diameter and mounted on two distinct devices, EMPI (Iwate University, Japan) and Chi-Kung (ANU, Australia). These experiments demonstrate that the DL and the supersonic ion beam are triggered when the ion Larmour radius in the source tube becomes smaller than the source tube radius, i.e., when the ions become magnetized in the source.

The authors would like to thank Professor M. Lieberman from the University of California at Berkeley for helpful discussion, P. Alexander from the ANU, and Y. Shida from Iwate University for their technical assistance. This work is partially supported by a Grant-in-Aid for Young Scientists (A, Grant No. 22684031), Japan. Part of this work is also supported by TEPCO Research Foundation.

${ }^{1}$ R. W. Boswell, E. Marsch, and C. Charles, Astrophys. J. 640, L199 (2006)

${ }^{2}$ C. Charles, J. Phys. D: Appl. Phys. 42, 163001 (2009)

${ }^{3}$ C. Charles, Plasma Sources Sci. Technol. 16, R1 (2007)

${ }^{4}$ M. A. Lieberman and C. Charles, Phys. Rev. Lett. 97, 045003 (2006).

${ }^{5}$ C. Charles, R. W. Boswell, R. Laine, and P. MacLellan, J. Phys. D: Appl. Phys. 41, 175213 (2008)

${ }^{6}$ S. C. Thakur, Z. Harvey, I. A. Biloiu, A. Hansen, R. A. Hardin, W. S. Przybysz, and E. E. Scime, Phys. Rev. Lett. 102, 035004 (2009).

${ }^{7}$ C. Charles and R. W. Boswell, Appl. Phys. Lett. 91, 201505 (2007).

${ }^{8}$ K. Takahashi, K. Oguni, H. Yamada, and T. Fujiwara, Phys. Plasmas 15 , 084501 (2008).

${ }^{9}$ C. Charles and R. W. Boswell, IEEE Trans. Plasma Sci. 36, 2141 (2008).

${ }^{10}$ V. F. Virko, Y. V. Virko, V. M. Slobodyan, and K. P. Shamrai, Plasma Sources Sci. Technol. 19, 015004 (2010).

${ }^{11}$ K. Takahashi, Y. Shida, and T. Fujiwara, Plasma Sources Sci. Technol. 19, 025004 (2010).

${ }^{12}$ A. Meige, R. W. Boswell, C. Charles, and M. M. Turner, Phys. Plasmas 12, 052317 (2005).

${ }^{13}$ K. Takahashi, Y. Shida, and T. Fujiwara, J. Plasma Fusion Res. SERIES 9, 381 (2010).

${ }^{14}$ C. Charles and R. W. Boswell, Appl. Phys. Lett. 82, 1356 (2003).

${ }^{15}$ A. M. Keesee, E. E. Scime, C. Charles, A. Meige, and R. W. Boswell, Phys. Plasmas 12, 093502 (2005). 